EGU2020-7976, updated on 21 Apr 2020

https://doi.org/10.5194/egusphere-egu2020-7976

EGU General Assembly 2020

(c) Author(s) 2020. This work is distributed under

the Creative Commons Attribution 4.0 License.

\title{
Rayleigh wave ellipticity measurements in the North Tanzanian Divergence (Eastern African Rift)
}

\author{
Laura Parisi ${ }^{1}$, Andrea Berbellini ${ }^{2}$, and P. Martin Mai ${ }^{1}$ \\ ${ }^{1}$ Physical Science and Engineering Division, King Abdullah University of Science and Technology, Thuwal, Saudi Arabia \\ (laura.parisi@kaust.edu.sa) \\ ${ }^{2}$ Istituto Nazionale di Geofisica e Vulcanologia, sezione di Bologna, via Donato Creti 12, 40128 Bologna, Italy
}

Rayleigh wave ellipticity depends, in theory, only on the Earth structure below a seismic station, offering the advantage of a "single-station" method to infer crustal properties. Therefore, ellipticity measurements can be used to construct pseudo 3-D shear velocity models of the earth structure using even seismic stations that did not record simultaneously.

Based on that, we carried-out ellipticity measurements by using teleseismic waveforms recorded by the OPS seismic network we deployed at the western flank of the North Tanzanian Divergence between June 2016 and May 2018, covering 17 sites. We then expanded our measurements on the waveforms recorded by the adjacent CRAFTI seismic network from January 2013 and December 2014, available on IRIS, which comprised more than 30 sites.

While the OPS network covers the transition between the Tanzania Craton and North Tanzanian Divergence, the CRAFTI network is entirely contained in the North Tanzanian Divergence. Therefore, the imaging that can be obtained by integrating the two asynchronous passive seismology experiments will help to better understand the dynamics of this segment of the eastern branch of the Eastern African Rift.

Preliminary results show heterogeneity structure that are in agreement with previous tomographic studies based on ambient noise cross-correlation and body-waves arrival-times. In regions where previous seismological studies are not available, results match the known geological structure of the transition between the Tanzanian Craton and the North Tanzanian Divergence. This demonstrates that measurements of ellipticity can be a useful and integrative tool for earth structure imaging, especially at the edges of the active rifts where the seismicity is scarce. 